\title{
Abstract: MeV ion scattering from thin Si single crystals: A novel approach to interface studies
}

L. C. Feldman, P. J. Silverman, and I. Stensgaard

Bell Laboratories, Murray Hill, New Jersey 07974

\section{N. Cheung}

Department of Applied Physics, California Institute of Technology, Pasadena, California 91125

(Received 13 September 1979)

PACS numbers: $68.20 .+\mathrm{t}, 79.20 . \mathrm{Nc}, 61.80 . \mathrm{Jh}, 61.80 . \mathrm{Mk}$

$\mathrm{MeV}$ ion scattering, in combination with channeling, provides a quantitative non-destructive method for determining the stoichiometry of amorphous layers on semiconductors and the reconstruction of the single crystal substrate. The interpretation and sensitivity of the technique depend on proper knowledge of the ion beam flux distribution and the thermal displacements of the atoms in the unreconstructed monolayers of the single crystal surface. We describe measurements of the scattering intensity from thin $(\sim 0.5 \mu \mathrm{m})$ Si crystals with thin oxides. The ion beam flux distribution is modified in channeling through such thin crystals so that the beam at the exit surface scatters primarily from atoms with large displacements from the atomic strings. The scattering spectrum from the beam exit side therefore displays a peak which represents only those channeled ions which have scattered from $\mathrm{Si}$ atoms in the oxide and from sufficiently displaced atoms in the reconstructed layers of the substrate. The intensity of this peak indicates that the native oxide is stoichiometric to within a few monolayers of the substrate, confirming the sharp interface previously reported. ${ }^{1}$ New information on the reconstruction at the Si interface is extracted and indicates that no more than two monolayers of Si are displaced substantially from their "bulk" positions. Possibilities for studies of metal-Si interfaces will also be discussed.

Acknowledgment: Work performed as a resident visitor at Bell Laboratories.

${ }^{1}$ L. C. Feldman, I. Stensgaard, P. J. Silverman, and T. E. Jackman in The Physics of $\mathrm{SiO}_{2}$ and Its Interfaces, edited by S. T. Pantelides (Pergamon, New York, 1978), p. 344. 